TECHNO: JURNAL PENELITIAN

Journal homepage: http://ejournal.unkhair.ac.id/index.php/Techno

Issue 09 Number 02 October 2020 DOI: http:/ / dx.doi.org/10.33387/tk.v9i2

\title{
Etnobotany in Customary Ceremony in Dayak Society, UUD Danum
}

\author{
Hendrikus Julung1, Benediktus Ege ${ }^{2}$ \\ ${ }^{1}$ Program Studi Pendidikan Biologi, STKIP Persada Khatulistiwa, Indonesia. Email: \\ henjulung@gmail.com \\ ${ }^{2}$ Program Studi Pendidikan Biologi, STKIP Persada Khatulistiwa, Indonesia. Email: \\ ama_tamo@yahoo.com
}

Received: $15-09-2020$

Accepted:16-10-2020

Available online: 07-11-2020

\begin{abstract}
The Dayak Uud Danum people still maintain the traditional ceremonies of birth, marriage, death, and traditional medicine. This tradition is carried out as a tribute to the cultural heritage of the ancestors. This tradition cannot be separated from the use of plants as media which have an important role. However, the effects of globalization can threaten the sustainability of these plants, so an inventory of their existence is needed. This study aims to identify various types of plants used in various customary traditions in the Dayak Uud Danum tribe in Kemangai Village, Ambalau District. The research method used is a survey method with data collection tools in the form of semi-structured interviews. The results showed that there were 6 families of plants used for traditional birth rituals, 3 families for traditional marriage, 5 families for traditional death rituals, and 35 families for traditional medicine. Processing plants for custom births by boiling, drinking, and eating. In a wedding ceremony, the processing of plants is eaten, wrapped, and wrapped. In traditional death rituals, plants are used to make coffins, and statues are symbols of respect for the deceased. As for traditional medicine, most plants are processed by boiling and drinking. The parts of the plants that are most widely used in the four traditional rituals of the Dayak Uud Danum are the leaves, roots, and stems.
\end{abstract}

Keyword: Ethnobotany, Customary Ceremony, Uud Danum

\begin{abstract}
ABSTRAK
Masyarakat Suku dayak Uud Danum masih mempertahankan tradisi upacara adat kelahiran, perkawinan, kematian, dan pengobatan tradisional. Tradisi tersebut dilakukan sebagai penghormatan terhadap warisan budaya dari para leluhur. Tradisi tersebut tidak lepas dari penggunaan tumbuhan sebagai media yang memiliki peranan penting. Namun pengaruh globalisasi dapat mengancam kelestarian tumbuhan tersebut, sehingga diperlukan inventarisasi terhadap keberadaannya. Penelitian ini bertujuan untuk mengidentifikasi berbagai jenis tumbuhan yang digunakan dalam berbagai tradisi adat pada masyarakat suku dayak Uud Danum di Desa kemangai, Kecamatan Ambalau. Metode penelitian yang digunakan adalah metode survei dengan alat pengumpulan data berupa wawancara semi terstruktur. Hasil penelitian menunjukkan bahwa terdapat 6 famili tumbuhan yang digunakan untuk ritual adat kelahiran, 3 famili untuk adat pernikahan, 5 famili untuk ritual adat kematian, dan 35 famili untuk pengobatan tradisional. Pengolahan tumbuhan untuk adat kelahiran dengan cara direbus, diminum dan dimakan. Dalam acara pernikahan pengolahan tumbuhan dengan cara dimakan, dikalungkan dan dililitkan. Ritual adat kematian, tumbuhan digunakan untuk membuat peti mati, dan patung sebagai simbol penghormatan bagi yang meninggal. Adapun dalam pengobatan tradisonal, tumbuhan paling banyak diolah dengan cara direbus dan diminum. Bagian tumbuhan yang paling banyak digunakan dalam keempat ritual adat pada suku dayak Uud Danum adalah daun, akar, dan batang.
\end{abstract}

Kata Kunci: Etnobotani, Upacara Adat, Uud Danum 


\section{TECHNO: Vol. 09 (02) Oktober 2020}

\section{INTRODUCTION}

West Kalimantan is one of the islands with the fourth largest area in Indonesia $(26.98 \%$ of the total area of Indonesia) and forests reaching 9,125,486 hectares (Sardana et al., 2011). The majority of the people who live in West Kalimantan are Dayaks. This is supported by the results of research by Takoy et al., (2013) that the people living around the forest are dominated by the Dayak tribe. The Dayak tribe in West Kalimantan consists of 151 ethnic groups (Alloy et al., 2008), one of which is the Dayak Uud Danum in the Ambalau District, Sintang District, West Kalimantan. The Dayak Uud Danum tribe has its own local wisdom to conserve forests and plants as a conservation effort. One form of local wisdom of the Dayak Uud Danum tribe is traditional knowledge about the use of plants in traditional ceremonies of birth, marriage, death, and traditional medicine. The use of plants in traditional ceremonies has become a culture, one of which is in traditional birth rituals (Rahyuni et al., 2013). Likewise, the use of plants for medicine by the Dayak tribe has been used since ancient times (Aryadi et al., 2014).

Based on previous research, several problems identified regarding the use of plants (1) every year in Indonesia have decreased the land area of primary forest annually to reach 47,600 ha per year. In 2012, the increase in degradation of Indonesia's annual primary forest loss was higher at 0.84 Mha, when compared to 0.46 Mha in Brazil (Margono et.al., 2014). (2) Forest conditions in West Kalimantan are forest degradation in West Kalimantan continues to increase every year due to the increasing area of land clearing for the development of industrial plantations (HTI), oil palm plantations, illegal logging, and shifting cultivation threatens the sustainability of germplasm, rare and endemic species that exist, especially plants that have potential as medicinal plants (Takoy et al., 2005; Setyawan, 2010). These various problems can threaten the preservation of the traditional knowledge of the Dayak Uud Danum tribe in utilizing plants and threaten the preservation of biodiversity in the surrounding environment.

Many efforts have been made to utilize and conserve biodiversity, starting from an inventory of its use, cultivation and preservation, interdisciplinary approaches, for example through ethnobotany studies, maintaining local wisdom, and conducting conservation. Ethnobotany has the potential to reveal the traditional knowledge system of a community or ethnic group regarding conservation and cultural biodiversity (Tapundu \& Anam, 2015). Local wisdom is very important for the community in adapting to nature and becoming a cultural heritage in utilizing and processing natural resources with knowledge, customary norms, and cultural values contained in the concept of community thinking (Nurdin \& Ng, 2013).

The general objective of this research is as a conservation effort based on local wisdom through ethnobotany studies of plants used in traditional ceremonies of birth, marriage, divorce, death, and traditional medicine for the Dayak Uud Danum tribe in Ambalau District, Sintang District, West Kalimantan Province. The specific objectives of this research are (1) Identifying various types of plants used in traditional ceremonies (2) Describing traditional knowledge (ethnobotany studies) of the use of plants in traditional ceremonies in the Dayak Uud Danum tribe (3) Describing how conservation efforts based on Local Wisdom in Dayak Uud Danum tribe (4) Describe the theoretical feasibility of an ethnobotany encyclopedia as a conservation training material for the Dayak Uud Danum tribe in Ambalau District, Sintang District, West Kalimantan Province. 


\section{MATERIALS AND METHOD}

The approach used in this research is descriptive qualitative. A qualitative approach is used because the data collected is descriptive data in the form of written and spoken words from the Dayak Uud Danum community regarding the use of various types of plants for traditional ceremonies as conservation efforts based on local wisdom. The research method used the survey method. The survey method is used to collect data and obtain an overview of the phenomena that occur and develop naturally in the Dayak Uud Danum people, in the sense that the research variables are not manipulated by the researcher. The data source in this study comes from the Dayak Uud Danum people who have knowledge of the plants used in various traditional ceremonies in the Dayak Uud Danum tribe. The information selection technique used in this preliminary observation is the purposive sampling method, namely the technique of selecting informants with certain considerations, in this case the person who knows best about ritual plants. The figures chosen through this method to be interviewed are the traditional chiefs and shamans. Through this initial observation, it is known that the data of prospective informants for the next stage are suitable to be interviewed based on the recommendations of the customary chief and the shaman (Sugiyono, 2007). After initial observations, the study was calculated based on data about ritual plants to the population by means of interviews (Martin, 1995). The selection of informants at the interview stage was carried out using the snowball sampling method. Snowball Sampling means that the selection of key informants is based on the previous informants' recommendations (Sugiyono, 2007). Respondents selected as informants included Temenggung, traditional community leaders and village heads, who saw things that were closely related to the research being carried out. Collecting data through semi-structured interviews with a number of respondents who were determined to reveal the various activities of the Dayak Uud Danum ethnic community including various events, concepts, perceptions, community organizations in utilizing various plants in traditional ceremonies. After the data is carried out, the plant specimens are taken directly at the location where they are grown, assisted by a key informant. Specimens are photographed and identified. Data from the ethnobotany research of plants used in the ceremonies of birth, marriage, divorce, death and traditional medicine in the Dayak Uud Danum tribe were analyzed by: (1) determining the species of each plant, (2) calculating the proportion of the family, the part used, processing methods and habitat. Research data on conservation efforts based on local wisdom were analyzed by following Mieles and Huberman (2007) which consisted of data, data reduction, data presentation and conclusion.

\section{RESULTS AND DISCUSSION}

The results of the field survey through interviews with the Dayak Uud Danum people obtained 7 species used in birth ceremonies, as presented in Table 1.

Table 1. Types of Plants used in the Dayak Uud Danum Birth Ceremony

\begin{tabular}{cllllll}
\hline No & $\begin{array}{c}\text { Indonesian Name } \\
\text { (Local Name) }\end{array}$ & \multicolumn{1}{c}{$\begin{array}{c}\text { Scientific } \\
\text { name }\end{array}$} & Family & $\begin{array}{c}\text { The } \\
\text { Utilized } \\
\text { Part }\end{array}$ & Benefits & Use \\
\hline 1 & Pinang (Kahat) & $\begin{array}{l}\text { Areca catechu } \\
\text { L }\end{array}$ & Arecaceae & Root & $\begin{array}{l}\text { the root aids } \\
\text { postpartum } \\
\text { recovery }\end{array}$ & $\begin{array}{l}\text { boiled and } \\
\text { drunk }\end{array}$ \\
\hline 2 & $\begin{array}{l}\text { Alang-alang } \\
\text { (Tingon) }\end{array}$ & $\begin{array}{l}\text { Imperata } \\
\text { cylindrical }\end{array}$ & Cyperaceae & Root & $\begin{array}{l}\text { Accelerate } \\
\text { postpartum } \\
\text { recovery }\end{array}$ & $\begin{array}{l}\text { Boiled, } \\
\text { drunk }\end{array}$ \\
\hline 3 & $\begin{array}{l}\text { Sabang Hijau } \\
\text { (Savang behijau) }\end{array}$ & $\begin{array}{l}\text { Cordyline } \\
\text { fruticosa } \mathrm{L}\end{array}$ & Asparagaceae & Leaf & $\begin{array}{l}\text { Accelerate } \\
\text { postpartum }\end{array}$ & $\begin{array}{l}\text { The leaves } \\
\text { are }\end{array}$ \\
\hline
\end{tabular}


TECHNO: Vol. 09 (02) Oktober 2020

\begin{tabular}{|c|c|c|c|c|c|c|}
\hline & & & & & recovery & $\begin{array}{l}\text { squeezed } \\
\text { and drunk }\end{array}$ \\
\hline 4 & $\begin{array}{l}\text { Nangka Belanda } \\
\text { (Nakak Beradak) }\end{array}$ & $\begin{array}{l}\text { Annona } \\
\text { muricata }\end{array}$ & Annonaceae & Root & $\begin{array}{l}\text { The roots are } \\
\text { for postpartum } \\
\text { recovery }\end{array}$ & $\begin{array}{l}\text { boiled and } \\
\text { drunk }\end{array}$ \\
\hline 5 & Kunyit (Henak) & $\begin{array}{l}\text { Curcuma } \\
\text { longa Linn }\end{array}$ & Zingiberaceae & Rhizome & $\begin{array}{l}\text { Accelerate } \\
\text { postpartum } \\
\text { wound healing }\end{array}$ & $\begin{array}{l}\text { Grated, } \\
\text { drunk }\end{array}$ \\
\hline 6 & Kedadai/Ara (Harak) & $\begin{array}{l}\text { Ficus } \\
\text { variegate }\end{array}$ & Moraceae & Leaf & $\begin{array}{l}\text { Smooth and } \\
\text { reproduce } \\
\text { breast milk }\end{array}$ & $\begin{array}{l}\text { Boiled, } \\
\text { Edible }\end{array}$ \\
\hline 7 & $\begin{array}{l}\text { Jahe Merah (liok } \\
\text { mangan) }\end{array}$ & $\begin{array}{l}\text { Zingiber } \\
\text { officinale Rosc }\end{array}$ & Zingiberaceae & Rhizome & $\begin{array}{l}\text { Increase } \\
\text { stamina and } \\
\text { postpartum } \\
\text { wound } \\
\text { recovery }\end{array}$ & $\begin{array}{l}\text { boiled and } \\
\text { drunk }\end{array}$ \\
\hline
\end{tabular}

Based on the data in Table 1, it is known that the plant families used for the birth ceremony include Zingiberaceae (2 species), Moraceae (1 species), Annonaceae (1 species), Asparagaceae (1 species), Cyperaceae (1 species) and Arecaceae (1 species). ). The parts of the plants used in traditional birth ceremonies are the roots, leaves, and rhizomes. The use of roots, leaves, and rhizomes is mostly used after giving birth with a traditional procession led by a traditional leader with the assistance of a shaman. Meanwhile, for plants used in wedding ceremonies, it can be seen in Table 2.

Table 2. Types of Plants used in the Dayak Uud Danum Wedding Ceremony

\begin{tabular}{|c|c|c|c|c|c|c|}
\hline No & $\begin{array}{l}\text { Indonesian Name } \\
\text { (Local Name) }\end{array}$ & Scientific name & Family & $\begin{array}{c}\text { The } \\
\text { Utilized } \\
\text { Part }\end{array}$ & Benefits & Use \\
\hline 1 & Pinang (Kahat) & Areca catechu L. & Arecaceae & Fruit & $\begin{array}{l}\text { So that the } \\
\text { bride and } \\
\text { groom } \\
\text { complement } \\
\text { each other in } \\
\text { married life }\end{array}$ & $\begin{array}{l}\text { Eaten by the } \\
\text { bride and } \\
\text { groom }\end{array}$ \\
\hline 2 & Sirih (lout) & Piper bettle L. & Piperaceae & Leaf & $\begin{array}{l}\text { Symbol of } \\
\text { mutual loyalty } \\
\text { in living } \\
\text { together }\end{array}$ & $\begin{array}{l}\text { Eaten by the } \\
\text { bride and } \\
\text { groom along } \\
\text { with betel nuts }\end{array}$ \\
\hline 3 & $\begin{array}{l}\text { Sabang Hijau } \\
\text { (sabang behijau) }\end{array}$ & $\begin{array}{l}\text { Cordyline } \\
\text { fruticosa } \\
\text { (L.) A.Chev. }\end{array}$ & Asparagaceae & $\begin{array}{l}\text { Leaf } \\
\text { (pucuk) }\end{array}$ & $\begin{array}{l}\text { Damn } \\
\text { repellent }\end{array}$ & $\begin{array}{l}\text { Planted as a } \\
\text { symbol of the } \\
\text { start of a life } \\
\text { together }\end{array}$ \\
\hline 4 & $\begin{array}{l}\text { Cocor Bebek (Somo } \\
\text { Morum) }\end{array}$ & $\begin{array}{l}\text { Bryophyllum } \\
\text { pinnatum (Lam) }\end{array}$ & Crassulaceae & Leaf & $\begin{array}{l}\text { Has benefits as } \\
\text { a symbol or } \\
\text { sign of life } \\
\text { that is always } \\
\text { growing and } \\
\text { alive }\end{array}$ & $\begin{array}{l}\text { The leaves are } \\
\text { taken and } \\
\text { crushed and } \\
\text { rubbed on the } \\
\text { heads of the } \\
\text { bride and } \\
\text { groom. }\end{array}$ \\
\hline 5 & $\begin{array}{l}\text { Rotan Segak (Uwi } \\
\text { Sohkok) }\end{array}$ & $\begin{array}{l}\text { Calamus caesius } \\
\text { Blume }\end{array}$ & Arecaceae & Stem & $\begin{array}{l}\text { Has the benefit } \\
\text { of being a } \\
\text { marker of a } \\
\text { long and } \\
\text { strong life }\end{array}$ & $\begin{array}{l}\text { Wrapped } \\
\text { around the } \\
\text { neck of the } \\
\text { bride and } \\
\text { groom }\end{array}$ \\
\hline
\end{tabular}


Julung, H., Benediktus E. 2020. Ethnobotany, Customary Ceremony, Uud Danum

\begin{tabular}{|c|c|c|c|c|c|c|}
\hline 6 & $\begin{array}{l}\text { Akar Bajakah } \\
\text { tengang (Talik } \\
\text { Togang) }\end{array}$ & $\begin{array}{l}\text { Spatholobus } \\
\text { Littoralis Hassk }\end{array}$ & Leguminosae & Stem & $\begin{array}{l}\text { Marriage cord } \\
\text { enhancer / } \\
\text { Spirit } \\
\text { amplifier }\end{array}$ & $\begin{array}{l}\text { Marriage cord } \\
\text { enhancer / } \\
\text { Spirit } \\
\text { amplifier }\end{array}$ \\
\hline
\end{tabular}

The data in Table 2 shows that there are 5 species with 3 families, where the Arecaceae family with 2 plant species, while the Leguminosae and Asparagaceae families each with 1 plant species used in marriage ceremonies in the Dayak Uud Danum community. The Dayak Uud Danum people still maintain the traditional wedding traditions very well. Plants used in traditional ceremonies have a meaning so that a married couple can maintain household life responsibly. The plants used are symbols and at the same time a reminder for the married couple. Furthermore, the plants used in death ceremonies in the Dayak Uud Danum tribe can be seen in Table 3.

Table 3. Types of Plants used in the Dayak Uud Danum Death Ceremony

\begin{tabular}{|c|c|c|c|c|c|c|}
\hline No & $\begin{array}{c}\text { Indonesian } \\
\text { Name (Local } \\
\text { Name) }\end{array}$ & $\begin{array}{l}\text { Scientific } \\
\text { name }\end{array}$ & Family & $\begin{array}{c}\text { The Utilized } \\
\text { Part }\end{array}$ & Benefits & Use \\
\hline 1 & $\begin{array}{l}\text { Tebelian/kayu } \\
\text { ulin (Tobolion) }\end{array}$ & $\begin{array}{l}\text { Eusideroxylon } \\
\text { zwageri Teijs } \\
\text { m. \& Binn }\end{array}$ & Lauraceae & $\begin{array}{l}\text { The meat of the } \\
\text { stem }\end{array}$ & $\begin{array}{l}\text { - As a material } \\
\text { for building } \\
\text { graves/tombs } \\
\text { - To make a } \\
\text { coffin } \\
\text { - To make a } \\
\text { statue } \\
\text { (Sopunduk) as } \\
\text { a symbol of } \\
\text { respect for the } \\
\text { deceased's } \\
\text { family }\end{array}$ & $\begin{array}{l}\text { Used to make } \\
\text { blocks, } \\
\text { battens, } \\
\text { rafters, boards, } \\
\text { roofs }\end{array}$ \\
\hline 2 & $\begin{array}{l}\text { Alang-alang } \\
\text { (Tingon) }\end{array}$ & $\begin{array}{l}\text { Imperata } \\
\text { cylindrica } \mathrm{Rae} \\
\text { usch. }\end{array}$ & Cyperaceae & $\begin{array}{l}\text { Roots, stems, } \\
\text { and leaves }\end{array}$ & $\begin{array}{l}\text { To spare from } \\
\text { bad things } \\
\text { (bad luck) }\end{array}$ & $\begin{array}{l}\text { Flicked to the } \\
\text { body area } \\
\text { (Mohpas) }\end{array}$ \\
\hline 3 & $\begin{array}{l}\text { Mengkirai (Kajuk } \\
\text { Batuk) }\end{array}$ & $\begin{array}{l}\text { Trema } \\
\text { orientalis (L.) } \\
\text { Blume }\end{array}$ & Cannabaceae & $\begin{array}{l}\text { The meat of the } \\
\text { stem }\end{array}$ & $\begin{array}{l}\text { As a material } \\
\text { for making } \\
\text { coffins, statues } \\
\text { of death }\end{array}$ & $\begin{array}{l}\text { Used to make } \\
\text { blocks, } \\
\text { battens, } \\
\text { rafters, boards }\end{array}$ \\
\hline 4 & Meranti (Rotang) & $\begin{array}{l}\text { Shorea } \\
\text { tumbuggaia } \\
\text { Roxb. }\end{array}$ & $\begin{array}{l}\text { Dipterocarpac } \\
\text { eae }\end{array}$ & $\begin{array}{l}\text { The meat of the } \\
\text { stem }\end{array}$ & $\begin{array}{l}\text { As a material } \\
\text { for making } \\
\text { coffins, death } \\
\text { statues, tomb } \\
\text { houses }\end{array}$ & $\begin{array}{l}\text { Used to make } \\
\text { blocks, } \\
\text { battens, } \\
\text { rafters, boards }\end{array}$ \\
\hline 5 & $\begin{array}{l}\text { Keladan } \\
\text { (Koladan) }\end{array}$ & $\begin{array}{l}\text { Dipterocarpus } \\
\text { gracilis } \\
\text { Blume. }\end{array}$ & $\begin{array}{l}\text { Dipterocarpac } \\
\text { eae }\end{array}$ & $\begin{array}{l}\text { The meat of the } \\
\text { stem }\end{array}$ & $\begin{array}{l}\text { As a material } \\
\text { for making } \\
\text { coffins, death } \\
\text { statues, tomb } \\
\text { houses }\end{array}$ & $\begin{array}{l}\text { Used to make } \\
\text { blocks, } \\
\text { battens, } \\
\text { rafters, boards }\end{array}$ \\
\hline 6 & Kempas (Opas) & $\begin{array}{l}\text { Koompassia } \\
\text { malaccensis } \\
\text { Benth. }\end{array}$ & Fabaceae & $\begin{array}{l}\text { The meat of the } \\
\text { stem }\end{array}$ & $\begin{array}{l}\text { As a material } \\
\text { for making } \\
\text { coffins, death } \\
\text { statues, tomb } \\
\text { houses }\end{array}$ & $\begin{array}{l}\text { As a material } \\
\text { for making } \\
\text { coffins, death } \\
\text { statues, tomb } \\
\text { houses }\end{array}$ \\
\hline
\end{tabular}

Based on Table 3, it is known that there are 6 species from 5 plant families that are used in death ceremonies in the Dayak Uud Danum tribe. There are 2 plant species in the 
Dipterocarpaceae family, while the Fabaceae, Cannabaceae, Cyperaceae, and Lauraceae families each have one species. The Dipterocarpaceae family is mostly used to make coffins, statues as a symbol of respect for people who have died due to plants in the family such as keladan (Dipterocarpus gracilis Blume) and meranti (Shorea tumbuggaia Roxb) are still found in the forest near the settlement of the Dayak tribe community, Uud Danum. This is in line with the results of research by Kuni et al. (2015) which states that the family that is most widely used as a building material is the Dipterocarpaceae family which has high wood quality. As for the plants used in traditional medicine, it can be seen in Table 4.

Table 4. Types of Plants used in Traditional Medicine of the Dayak Uud Danum Tribe

\begin{tabular}{|c|c|c|c|c|c|c|}
\hline No & $\begin{array}{c}\text { Indonesian Name } \\
\text { (Local Name) }\end{array}$ & $\begin{array}{l}\text { Scientific } \\
\text { name }\end{array}$ & Family & $\begin{array}{c}\text { The Utilized } \\
\text { Part }\end{array}$ & Benefits & Use \\
\hline 1 & $\begin{array}{l}\text { Alang-alang } \\
\text { (Tingon) }\end{array}$ & $\begin{array}{l}\text { Imperata } \\
\text { cylindrica } \\
\text { Raeusch. }\end{array}$ & Cyperaceae & Root (Uhat) & $\begin{array}{l}\text { Launching } \\
\text { defecation, } \\
\text { treating } \\
\text { pain when } \\
\text { urinating }\end{array}$ & $\begin{array}{l}\text { Boiled, } \\
\text { drunk }\end{array}$ \\
\hline 2 & $\begin{array}{l}\text { Bawang Lembit } \\
\text { (Bawang Lombak) }\end{array}$ & $\begin{array}{l}\text { Eleutherine } \\
\text { americana } \\
\text { Merr }\end{array}$ & Iridaceae & Bulbs & $\begin{array}{l}\text { Treating } \\
\text { Tension, } \\
\text { Rheumatis } \\
\text { m, Tired, } \\
\text { Blood } \\
\text { Crack }\end{array}$ & $\begin{array}{l}\text { Boiled, } \\
\text { drunk }\end{array}$ \\
\hline 3 & $\begin{array}{l}\text { Bawang putih } \\
\text { (Bawang Putik) }\end{array}$ & $\begin{array}{l}\text { Allium } \\
\text { sativum } \\
\text { Linn. }\end{array}$ & Alliaceae & Bulbs & $\begin{array}{l}\text { Treating } \\
\text { Ulcer and } \\
\text { Treating } \\
\text { Hypertensi } \\
\text { on }\end{array}$ & $\begin{array}{l}\text { Burned, then } \\
\text { chewed }\end{array}$ \\
\hline 4 & $\begin{array}{l}\text { Bambu kuning } \\
\text { (Tolung Kuning) }\end{array}$ & $\begin{array}{l}\text { Bambusa } \\
\text { vulgaris } \text { Sch } \\
\text { rad }\end{array}$ & Poaceae & Root & $\begin{array}{l}\text { Treat } \\
\text { hepatitis }\end{array}$ & $\begin{array}{l}\text { Boiled, } \\
\text { drunk }\end{array}$ \\
\hline 5 & $\begin{array}{l}\text { Paku Pedang/paku } \\
\text { Harupat (Pakuk } \\
\text { Bahkai) }\end{array}$ & $\begin{array}{l}\text { Polypodium } \\
\text { verrucosum } \\
\text { (Hook) } \\
\text { Wall. }\end{array}$ & Polypodiaceae & Shoots & $\begin{array}{l}\text { Increase } \\
\text { breast milk }\end{array}$ & $\begin{array}{l}\text { Boiled, } \\
\text { drunk }\end{array}$ \\
\hline 6 & $\begin{array}{l}\text { Belimbing Wuluh } \\
\text { (Belimbing Tinjuk) }\end{array}$ & $\begin{array}{l}\text { Averrhoa } \\
\text { bilimbi Linn. }\end{array}$ & Oxalidaceae & Shoots & $\begin{array}{l}\text { Treating } \\
\text { Hypertensi } \\
\text { on } \\
\end{array}$ & $\begin{array}{l}\text { Boiled, } \\
\text { drunk }\end{array}$ \\
\hline 7 & $\begin{array}{l}\text { Daun Salam } \\
\text { (ohang) }\end{array}$ & $\begin{array}{l}\text { Syzygium } \\
\text { polyanthum } \\
\text { (Wight) } \\
\text { Walp } \\
\end{array}$ & Myrtaceae & Leaf & $\begin{array}{l}\text { Treat } \\
\text { smallpox }\end{array}$ & $\begin{array}{l}\text { Pounded, } \\
\text { smeared }\end{array}$ \\
\hline 8 & Cangkok (cangkok) & $\begin{array}{l}\text { Sauropus } \\
\text { androgynous } \\
\text { (L.) Merr }\end{array}$ & Euphorbiaceae & Leaf & $\begin{array}{l}\text { Treating } \\
\text { Pain in the } \\
\text { ribs }\end{array}$ & $\begin{array}{l}\text { Pounded, } \\
\text { smeared }\end{array}$ \\
\hline 9 & $\begin{array}{l}\text { Cocor Bebek } \\
\text { (Somo Morum) }\end{array}$ & $\begin{array}{l}\text { Bryophylum } \\
\text { pinnatum } \\
\text { (Lam.) }\end{array}$ & Crassulaceae & Leaf & $\begin{array}{l}\text { Lowering } \\
\text { Heat }\end{array}$ & $\begin{array}{l}\text { When } \\
\text { growing, } \\
\text { affixed/com } \\
\text { pressed on } \\
\text { the forehead }\end{array}$ \\
\hline 10 & $\begin{array}{l}\text { Temulawak Putih } \\
\text { (Lokuing) }\end{array}$ & $\begin{array}{l}\text { Curcuma } \\
\text { domestica } \\
\text { (Val.) }\end{array}$ & Zingiberaceae & Rhizome & $\begin{array}{l}\text { Treating } \\
\text { blisters on } \\
\text { the skin as } \\
\text { cut }\end{array}$ & $\begin{array}{l}\text { Pounded, } \\
\text { smeared }\end{array}$ \\
\hline
\end{tabular}




\begin{tabular}{|c|c|c|c|c|c|c|}
\hline 11 & $\begin{array}{l}\text { Gelinggang } \\
\text { (Linggang) }\end{array}$ & $\begin{array}{l}\text { Cassia alata } \\
\text { (L.) }\end{array}$ & Caesalpiniaceae & Leaf & $\begin{array}{l}\text { Treat } \\
\text { ringworm }\end{array}$ & $\begin{array}{l}\text { Pounded, } \\
\text { smeared }\end{array}$ \\
\hline 12 & $\begin{array}{l}\text { Jahe Merah (liok } \\
\text { mangan) }\end{array}$ & $\begin{array}{l}\text { Zingiber } \\
\text { officinale } \\
\text { (Rosc) }\end{array}$ & Zingiberaceae & Rhizome & $\begin{array}{l}\text { Increase } \\
\text { stamina }\end{array}$ & $\begin{array}{l}\text { Boiled, } \\
\text { drunk }\end{array}$ \\
\hline 13 & $\begin{array}{l}\text { Jambu Biji (Jambu } \\
\text { Bojah) }\end{array}$ & $\begin{array}{l}\text { Psidium } \\
\text { guajava (L.) }\end{array}$ & Myrtaceae & Leaf & $\begin{array}{l}\text { Treat } \\
\text { stomach } \\
\text { pain }\end{array}$ & $\begin{array}{l}\text { Boiled, } \\
\text { drunk }\end{array}$ \\
\hline 14 & $\begin{array}{l}\text { Pisang Nipah } \\
\text { (Putik ipah) }\end{array}$ & $\begin{array}{l}\text { Musa } \\
\text { paradisiaca }\end{array}$ & Musaceae & $\begin{array}{l}\text { Banana } \\
\text { flower/heart }\end{array}$ & $\begin{array}{l}\text { Increase } \\
\text { breast milk }\end{array}$ & $\begin{array}{l}\text { Boiled, } \\
\text { drunk }\end{array}$ \\
\hline 15 & $\begin{array}{l}\text { Jerangau } \\
\text { (Samorongok) }\end{array}$ & $\begin{array}{l}\text { Acorus } \\
\text { calamus (L.) }\end{array}$ & Acoraceae & Rhizome, Stems & $\begin{array}{l}\text { Treating } \\
\text { coughs, } \\
\text { abdominal } \\
\text { pain, } \\
\text { bowel } \\
\text { movement } \\
\text { s } \\
\text { accompani } \\
\text { ed by } \\
\text { blood }\end{array}$ & $\begin{array}{l}\text { Smoothed, } \\
\text { smeared/boil } \\
\text { ed, and } \\
\text { drunk }\end{array}$ \\
\hline 16 & $\begin{array}{l}\text { Benalu (kajuk } \\
\text { tukun) }\end{array}$ & $\begin{array}{l}\text { Dendropthoe } \\
\text { petandra } \\
\text { (L.) Miq }\end{array}$ & Loranthaceae & Twig & $\begin{array}{l}\text { Defeating } \\
\text { opponents } \\
\text { in battle }\end{array}$ & $\begin{array}{l}\text { tucked in the } \\
\text { body }\end{array}$ \\
\hline 17 & $\begin{array}{l}\text { Kayu kempunan } \\
\text { (kayu dua warna) }\end{array}$ & $\begin{array}{l}\text { Excoecaria } \\
\text { cochinchine } \\
\text { nsis }\end{array}$ & Euphorbiaceae & Leaf & $\begin{array}{l}\text { magic } \\
\text { element- } \\
\text { Treating } \\
\text { inflammati } \\
\text { on (pain } \\
\text { due to } \\
\text { forgetting } \\
\text { to eat } \\
\text { something, } \\
\text { then illness } \\
\text { occurs) }\end{array}$ & $\begin{array}{l}\text { Boiled, } \\
\text { drunk }\end{array}$ \\
\hline 18 & $\begin{array}{l}\text { Kemunting } \\
\text { (Kolomunting) }\end{array}$ & $\begin{array}{l}\text { Rhodomyrtu } \\
\text { s } \\
\text { tomentosa } \\
\text { (Aiton) } \\
\text { Hassk }\end{array}$ & Myrtaceae & Leaf & $\begin{array}{l}\text { Treat: } \\
\text { Stomach } \\
\text { pain, cover } \\
\text { wounds, } \\
\text { stop } \\
\text { bleeding }\end{array}$ & $\begin{array}{l}\text { The extract } \\
\text { is boiled, } \\
\text { drunk, } \\
\text { crushed, and } \\
\text { then dropped } \\
\text { on the } \\
\text { wound }\end{array}$ \\
\hline 19 & Kunyit (hennak) & $\begin{array}{l}\text { Curcuma } \\
\text { longa (Linn. } \\
\text { ) }\end{array}$ & Zingiberaceae & Rhizome & $\begin{array}{l}\text { Treat ulcer } \\
\text { disease }\end{array}$ & $\begin{array}{l}\text { Grated, } \\
\text { drunk }\end{array}$ \\
\hline 20 & $\begin{array}{l}\text { Kedadai/Ara } \\
\text { (Harak) }\end{array}$ & $\begin{array}{l}\text { Ficus } \\
\text { variegata } \\
\text { (Blume) }\end{array}$ & Moraceae & Leaf & $\begin{array}{l}\text { Smooth } \\
\text { and } \\
\text { reproduce } \\
\text { breast milk }\end{array}$ & $\begin{array}{l}\text { Boiled, } \\
\text { Edible }\end{array}$ \\
\hline 21 & $\begin{array}{l}\text { Kelapa Kuning } \\
\text { (Oyuh Kuning) }\end{array}$ & $\begin{array}{l}\text { Cocos } \\
\text { nucifera (L.) }\end{array}$ & Arecaceae & Root & $\begin{array}{l}\text { Assist in } \\
\text { postpartum } \\
\text { recovery }\end{array}$ & $\begin{array}{l}\text { Boiled, } \\
\text { drunk }\end{array}$ \\
\hline 22 & Langsat (Lihat) & $\begin{array}{l}\text { Lansium } \\
\text { domesticum } \\
\text { Corr }\end{array}$ & Meliaceae & Skin stems & $\begin{array}{l}\text { Treating } \\
\text { malaria, } \\
\text { headaches }\end{array}$ & $\begin{array}{l}\text { Boiled, } \\
\text { drunk }\end{array}$ \\
\hline 23 & Lengkuas (Rokuah) & $\begin{array}{l}\text { Alpinia } \\
\text { galanga }(\mathrm{L} .)\end{array}$ & Zingiberaceae & Rhizome & $\begin{array}{l}\text { Treat } \\
\text { ringworm } \\
\text { and scabies }\end{array}$ & $\begin{array}{l}\text { Pounded, } \\
\text { smeared }\end{array}$ \\
\hline 24 & Lengkeng & Dimocarpus & Sapindaceae & & Defecation & Scrape, the \\
\hline
\end{tabular}


TECHNO: Vol. 09 (02) Oktober 2020

\begin{tabular}{|c|c|c|c|c|c|c|}
\hline & (Tekohih) & longan Lour & & Epidermis & $\begin{array}{l}\text { accompani } \\
\text { ed by } \\
\text { blood and } \\
\text { mucus }\end{array}$ & $\begin{array}{l}\text { water is } \\
\text { drunk }\end{array}$ \\
\hline 25 & Pare (Priak) & $\begin{array}{l}\text { Momordica } \\
\text { charantia } \\
\text { (L.) }\end{array}$ & Cucurbitaceae & Leaf & $\begin{array}{l}\text { Treat } \\
\text { scabies }\end{array}$ & $\begin{array}{l}\text { Pounded, } \\
\text { smeared }\end{array}$ \\
\hline 26 & $\begin{array}{l}\text { Kasembung } \\
\text { (Momung) }\end{array}$ & $\begin{array}{l}\text { Blumea } \\
\text { balsamifera } \\
\text { (L.) }\end{array}$ & Compositae & Leaf & Treat fever & $\begin{array}{l}\text { Heated, } \\
\text { Sticked }\end{array}$ \\
\hline 27 & $\begin{array}{l}\text { Mengkudu } \\
\text { (Robonau) }\end{array}$ & $\begin{array}{l}\text { Morinda } \\
\text { citrifolia (L). }\end{array}$ & Rubiaceae & Root, Leaf & $\begin{array}{l}\text { Treating } \\
\text { Hypertensi } \\
\text { on }\end{array}$ & $\begin{array}{l}\text { Boiled, } \\
\text { drunk }\end{array}$ \\
\hline 28 & $\begin{array}{l}\text { Ciplukan } \\
\text { (korohtup) }\end{array}$ & $\begin{array}{l}\text { Physalis } \\
\text { peruviana } \\
\text { (L.) }\end{array}$ & Solanaceae & Root & $\begin{array}{l}\text { Light } \\
\text { stroke, } \\
\text { rheumatis } \\
\text { m }\end{array}$ & $\begin{array}{l}\text { Boiled, } \\
\text { drunk }\end{array}$ \\
\hline 29 & $\begin{array}{l}\text { Nangka Belanda } \\
\text { (Nakak Boranak) }\end{array}$ & $\begin{array}{l}\text { Annona } \\
\text { muricata } \\
\text { (L.) }\end{array}$ & Annonaceae & Leaf, Root & $\begin{array}{l}\text { Treating } \\
\text { tension and } \\
\text { gout, Its } \\
\text { roots are } \\
\text { for } \\
\text { postpartum } \\
\text { recovery }\end{array}$ & $\begin{array}{l}\text { Boiled, } \\
\text { drunk }\end{array}$ \\
\hline 30 & $\begin{array}{l}\text { Ngkeleban } \\
\text { (Kolopopak) }\end{array}$ & $\begin{array}{l}\text { Vitex } \\
\text { pinnata }(\mathrm{L} .)\end{array}$ & Verbenaceae & Shoots & $\begin{array}{l}\text { Treating } \\
\text { dysentery } \\
\text { and } \\
\text { vomiting }\end{array}$ & $\begin{array}{l}\text { Boiled, } \\
\text { drunk }\end{array}$ \\
\hline 31 & $\begin{array}{l}\text { Padi /Beras } \\
\text { (Paroi/Bajoh) }\end{array}$ & $\begin{array}{l}\text { Oryza sativa } \\
\text { (L.) }\end{array}$ & Poaceae & Seed & $\begin{array}{l}\text { Smooth } \\
\text { skin }\end{array}$ & $\begin{array}{l}\text { Pounded, } \\
\text { smeared }\end{array}$ \\
\hline 32 & $\begin{array}{l}\text { Pasak Bumi } \\
\text { (sulungsio) }\end{array}$ & $\begin{array}{l}\text { Eurycoma } \\
\text { longifolia } \\
\text { Jack. }\end{array}$ & Simaroubaceae & Root, Leaf & $\begin{array}{l}\text { Treating } \\
\text { malaria } \\
\text { pain, } \\
\text { Treating } \\
\text { Impotent }\end{array}$ & $\begin{array}{l}\text { Boiled, } \\
\text { drunk }\end{array}$ \\
\hline 33 & $\begin{array}{l}\text { Pelusur (Pelusur } \\
\text { Penganon/Kajuk } \\
\text { Randui) }\end{array}$ & $\begin{array}{l}\text { Alchornea } \\
\text { latifolia } \mathrm{Sw} .\end{array}$ & Euphorbiaceae & Leaf & $\begin{array}{l}\text { As a } \\
\text { stimulant } \\
\text { to facilitate } \\
\text { labor }\end{array}$ & $\begin{array}{l}\text { Boiled, } \\
\text { drunk }\end{array}$ \\
\hline 34 & $\begin{array}{l}\text { Pepaya (Buah } \\
\text { Tokajuk) }\end{array}$ & $\begin{array}{l}\text { Carica } \\
\text { papaya }(\mathrm{L} .)\end{array}$ & Caricaceae & Leaf, Rind & $\begin{array}{l}\text { Treating } \\
\text { fever, } \\
\text { worms, } \\
\text { toothache } \\
\text { and } \\
\text { headaches, } \\
\text { malaria }\end{array}$ & $\begin{array}{l}\text { Boiled, } \\
\text { drunk }\end{array}$ \\
\hline 35 & Pinang (Kahat) & $\begin{array}{l}\text { Areca } \\
\text { catechu (L.) }\end{array}$ & Arecaceae & Fruit, Root & $\begin{array}{l}\text { Treating } \\
\text { ulcers, the } \\
\text { roots help } \\
\text { postpartum } \\
\text { recovery }\end{array}$ & $\begin{array}{l}\text { Pounded and } \\
\text { smeared, } \\
\text { boiled and } \\
\text { drunk }\end{array}$ \\
\hline 36 & $\begin{array}{l}\text { Putri malu (kupai } \\
\text { nunduk) }\end{array}$ & $\begin{array}{l}\text { Mimosa } \\
\text { pudica }(\mathrm{L} .)\end{array}$ & Mimosaceae & $\begin{array}{l}\text { All parts of the } \\
\text { plant }\end{array}$ & $\begin{array}{l}\text { Used to } \\
\text { subdue an } \\
\text { opponent } \\
\text { (battle) }\end{array}$ & $\begin{array}{l}\text { Tuck on the } \\
\text { body }\end{array}$ \\
\hline
\end{tabular}




\begin{tabular}{|c|c|c|c|c|c|c|}
\hline 37 & $\begin{array}{l}\text { Rumput Belanda } \\
\text { (Uruk Borandak) }\end{array}$ & $\begin{array}{l}\text { Pennisetum } \\
\text { purpureum } \\
\text { Schumach. }\end{array}$ & Poaceae & Leaf & $\begin{array}{l}\text { Treat } \\
\text { wounds } \\
\text { and stop } \\
\text { bleeding } \\
\text { from } \\
\text { external } \\
\text { wounds }\end{array}$ & $\begin{array}{l}\text { Pounded, } \\
\text { Sticked }\end{array}$ \\
\hline 38 & $\begin{array}{l}\text { Sabang Hijau } \\
\text { (Savang bohijau) }\end{array}$ & $\begin{array}{l}\text { Cordyline } \\
\text { fruticosa } \\
\text { (L.) }\end{array}$ & Asparagaceae & Shoots & $\begin{array}{l}\text { Treating } \\
\text { wind } \\
\text { sitting (hit } \\
\text { by ghost } \\
\text { chopsticks) }\end{array}$ & $\begin{array}{l}\text { The water is } \\
\text { drunk at the } \\
\text { shoots }\end{array}$ \\
\hline 39 & $\begin{array}{l}\text { Sahang (Sahang } \\
\text { masih) }\end{array}$ & $\begin{array}{l}\text { Piper } \\
\text { nigrum (L.) }\end{array}$ & Piperaceae & Seed & $\begin{array}{l}\text { Treating } \\
\text { colds }\end{array}$ & $\begin{array}{l}\text { Treating } \\
\text { colds }\end{array}$ \\
\hline 40 & Serai (Sodorai) & $\begin{array}{l}\text { Cymbopogo } \\
n \text { citratus } \\
\text { (DC.) Stapf }\end{array}$ & Poaceae & $\begin{array}{l}\text { Root, Stems, } \\
\text { Leaf }\end{array}$ & $\begin{array}{l}\text { Treating } \\
\text { coughs, } \\
\text { colds }\end{array}$ & $\begin{array}{l}\text { Boiled and } \\
\text { drunk when } \\
\text { warm }\end{array}$ \\
\hline 41 & $\begin{array}{l}\text { Selasih (bungok } \\
\text { talan) }\end{array}$ & $\begin{array}{l}\text { Ocimum } \\
\text { basilicum } \\
\text { (L.) }\end{array}$ & Lamiaceae & Fruit & $\begin{array}{l}\text { Treat heat } \\
\text { in / fever }\end{array}$ & $\begin{array}{l}\text { Made a } \\
\text { drink }\end{array}$ \\
\hline 42 & Sengkubak (Sokai) & $\begin{array}{l}\text { Pycnarrhena } \\
\text { cauliflora } \\
\text { (Miers.) } \\
\text { Diels } \\
\end{array}$ & $\begin{array}{l}\text { Menispermacea } \\
e\end{array}$ & Leaf & Treat fever & $\begin{array}{l}\text { Used for } \\
\text { bathing }\end{array}$ \\
\hline 43 & Simpur (Sipur) & $\begin{array}{l}\text { Dillenia } \\
\text { philippinensi } \\
s \text { Rolfe. }\end{array}$ & Dilleniaceae & Leaf & $\begin{array}{l}\text { Restoratio } \\
\mathrm{n} \text { of energy } \\
\text { after } \\
\text { childbirth }\end{array}$ & $\begin{array}{l}\text { Boiled, } \\
\text { drunk }\end{array}$ \\
\hline 44 & Sirih (Lout) & $\begin{array}{l}\text { Piper bettle } \\
\text { (L.) }\end{array}$ & Piperaceae & Leaf & $\begin{array}{l}\text { Treating } \\
\text { blurred } \\
\text { eyes, } \\
\text { vaginal } \\
\text { discharge }\end{array}$ & $\begin{array}{l}\text { Boiled, } \\
\text { drunk }\end{array}$ \\
\hline 45 & $\begin{array}{l}\text { Sungsung/Kembang } \\
\text { Sepatu (Bunga } \\
\text { sopahtuk) }\end{array}$ & $\begin{array}{l}\text { Hibiscus } \\
\text { rosa-sinensis } \\
\text { (L.) }\end{array}$ & Malvaceae & Leaf & $\begin{array}{l}\text { Treat } \\
\text { swelling }\end{array}$ & $\begin{array}{l}\text { Pounded, } \\
\text { smeared }\end{array}$ \\
\hline 46 & Tebelian (Tobolion) & $\begin{array}{l}\text { Eusideroxylo } \\
n \text { zwageri } \\
\text { Teijsm. \& } \\
\text { Binn. } \\
\end{array}$ & Lauraceae & Stems & $\begin{array}{l}\text { Treating } \\
\text { Pain in the } \\
\text { ribs }\end{array}$ & $\begin{array}{l}\text { Burnt (take } \\
\text { ashes), } \\
\text { smeared }\end{array}$ \\
\hline 47 & $\begin{array}{l}\text { Tengkawang } \\
\text { (Avang) }\end{array}$ & $\begin{array}{l}\text { Shorea } \\
\text { stenoptera } \\
\text { Burck }\end{array}$ & $\begin{array}{l}\text { Dipterocarpacea } \\
\text { e }\end{array}$ & Fruit & $\begin{array}{l}\text { Treat } \\
\text { ulcers on } \\
\text { the outer } \\
\text { skin }\end{array}$ & $\begin{array}{l}\text { Burned, } \\
\text { pounded, } \\
\text { and pasted }\end{array}$ \\
\hline
\end{tabular}

The data in Table 4 shows that there are 47 species from 35 plant families used in traditional medicine in the Uud Danum Dayak tribe community. Plant families that are widely used in traditional medicine include Poaceae (4 species), Zingiberaceae (4 species), Myrtaceae (3 species), Euphorbiaceae (3 species), Arecaceae (2 species), and Piperaceae (2 species). While 29 other families each with 1 species. Poaceae is a plant that is widely cultivated or grows wild on various types of soil, such as lemongrass (Cymbopogon citratus), rice (Oryza sativa), Dutch grass (Pennisetum purpureum), and yellow bamboo (Bambusa vulgaris). The high use of plants from the Zingiberaceae family is due to Many people have cultivated it and it is usually planted in house gardens with the use of it as a medicine as well as a cooking spice and its economic value. The results of interviews conducted with Dayak community leaders, Uud Danum, in general, people use plants as traditional processed medicines. This is done before the sick 


\section{TECHNO: Vol. 09 (02) Oktober 2020}

patient is given further medical assistance at the health center. Traditional or natural treatment is more popular because it is cheaper and has minimal side effects compared to using modern medicines or chemical drugs (Kuni et al., 2015).

Furthermore, the part of the plant that is widely used in the traditional medicine of the Dayak Uud Danum tribe, namely leaves. The results of the interview indicated that leaves were used more in medicine because they were easier to obtain and did not damage plants and were easy to process. This is because it is easy to obtain and the leaves accumulate secondary metabolites that are useful as drugs (Cavoski et al., 2011) such as flavonoids (Van Wyk et al., 2002; Van Wyk \& Wink, 2004; John, 2008; Dewick, 2009; Ahmat et al., 2015), saponins (Lajuck, 2012), tannins (Aiyelaagbe et al., 2008), alkaloids (Ting et al., 2010; Charyadie, 2014; Lee et al., 2014), morphine (Dewick, 2009), shikonin, vinblastine vincristine, ajmalicine (Mariska, 2013) and essential oils (Patimah, 2010; Kabera et al., 2014). This chemical compound has medicinal properties to treat various types of diseases (Septiatin, 2008). Leaves are used because they are easier to obtain whenever people need them, and can be used to treat internal and external diseases (Maryadi, 2012). This is also supported by the results of research by Efremila et al. (2015) whereof the 50 plants, the most widely used part was leaves by $30 \%$.

Based on the processing method in utilizing medicinal plants, the Dayak Uud Danum people still do it traditionally. The processing of plants as medicine includes boiling, crushing, drinking, sticking, smearing, heating, burning, beating, pounding, and being used in bathing. Of all the processing methods, the method of boiling and drinking is the most widely used by the Dayak Uud Danum people. This is because the two methods are considered the easiest, most accurate, and quick to feel the medicinal properties. This is in line with the research of Supiandi, et al. (2019) which states that traditional medicine processing carried out by the Dayak village community is by boiling and drinking it.

\section{CONCLUSION}

The results of this study reported the number of plants used in traditional birth rituals as many as 7 species in 6 families. The number of plants for traditional marriage ceremonies is 5 species in 3 families. There are 6 species of plants used in the death ceremony in 5 families. There are 47 species of plants used in traditional medicinal rituals in 35 families. Parts of plant organs used were: 14 types of root leaves, 14 types of stems, 32 types of leaves, 5 types of fruit, 1 type of flower, 2 types of seeds, 7 types of rhizome, and 2 types of tubers. The use of ritual plants for the Dayak Uud Danum tribe is by pounding, grating, boiling, burning, crushing, heating, drinking, eating, tucking, sticking, wearing, flapping, smearing, planting and used as building materials. The Dayak Uud Danum people believe that human existence cannot be separated from nature. Nature has had a lot of influence in the customary traditions of the Dayak Uud Danum tribe. For the Dayak Uud Danum people, nature in particular the various types of plants that exist is a gift that must be preserved and preserved. Through these various customary traditions, the Dayak people pass their culture to their descendants to this day.

\section{ACKNOWLEDGMENTS}

Our gratitude goes to the Directorate of Research and Community Service, Ministry of Research and Technology/National Research and Innovation Agency No. SPPK: 087/SP2H/ AMD/LT/DRPM/2020 and Research Contract Number: 02/L-2/Research. STKIPPK/III/2020. Our gratitude also goes to the Chairperson of the Sintang Nation's National Education Agency Association, the Head of STKIP Persada Khatulistiwa Sintang, the Head of Nanga Kemangai Village, Traditional Management, Community Figures, and all informants 
who have provided assistance in the form of both technical and non-technical, directly or indirectly related with all research activities carried out in Nanga Kemangai Village, Ambalau District, Sintang District.

\section{REFERENCES}

Ahmad, A., Kaleem, M., Ahmed, Z., and Shafiq, H. 2015. Therapeutic potential of flavonoids and their mechanism of action against microbial and viral infections-A review. Food Research International, $77(2), 221-235$.

Aiyelaagbe, O. O., B. J. Oguntuase, B. D. Arimah, and B. A. Adeniyi, 2008. The Antimicrobial Activity of Jatropha multifida Extracts and Chromatographic Fractions Against SexuaEy Transmitted Infections. The University of Ibadan. Nigeria. Journal of Medical Science. 8 (2): 143-147.

Alloy S, Albertus, Yovinus and Istiyani CP. 2008. Peta Keberagaman Subsuku Dayak di Kalimantan Barat (Figure of Subethnics Dayaknese in West Kalimantan). Dayakologi Institute. Pontianak. Indonesia.

Aryadi, M., Fithria, A., Susilawati, Fatria. 2014. Kearifan Lokal Masyarakat Dayak Terhadap Tumbuhan.

Cavoski, I., Caboni, P., and Miano, T. 2011. Natural pesticides and future perspectives. InMargarita Stoytcheva (Eds.), Pesticides in the Modern World - Pesticides Use and Management, (pp. 169-190). Rijeka: InTech Europe.

Charyadie, F. L., S. Adi dan S. R. Parwati, 2014. Daya Hambat Ekstrak Daun Alpukat (Persea americana, Mill.) terhadap Pertumbuhan Enterococcus faecalis. Universitas Hang Tuah. Surabaya. 8 (1).

Dewick, P.M. 2009. Medicinal Natural Products: A Biosynthetic Approach, 3rd Edition. West Sussex, UK: John Wiley \& Sons, Ltd.

Efremila, Evy Wardenaar, Lolyta Sisillia. 2015. Studi Etnobotani Tumbuhan Obat Oleh Etnis Suku Dayak Di Desa Kayu Tanam Kecamatan Mandor Kabupaten Landak. Jurnal Hutan Lestari Vol. 3 (2) : $234-246$.

John, A., O., Emmanuel, and D. H. C. Witness, 2008. Antidiarrhoeal Activity of Psidium guajava L. Linn. (Myrtaceae) Leaf Aqueous Extracts in Rodents. Journal Smooth Muscle Res. 44(6):195-207.

Lajuck, P., 2012. Ekstrak Daun Salam (Eugenia poliantha) Lebih Efektif Menurunkan Kadar Kolesterol Total dan LDL Dibandingkan Statin pada Penderita Dislipidemia. Universitas Udayana. Denpasar.

Lee, S.T., Welch, K.D., Panter, K.E., Gardner, D.R., Garrossian, M., and Chang, C.T. 2014. Cyclopamine: From Cyclops Lambs to Cancer Treatment. Journal of Agriculture and Food Chemistry, 62(30),73557362.

Kabera, J.N., Semana, E., Mussa, A.R., and He, X. 2014. Plant Secondary Metabolites: Biosynthesis, Classification, Function, and Pharmacological Properties. Journal of Pharmacy and Pharmacology, 2, 377-392.

Kuni, B.E.,Gusti, H., Idham. (2015).Etnobotani Masyarakat Suku Dayak Kerabat Di Desa Tapang Perodah Kecamatan Sekadau Hulu Kabupaten Sekadau. Jurnal Hutan Lestari Vol. 2 (3): 383-400

Mariska, I. 2013. Metabolit Sekunder: Jalur pembentukan dan kegunaannya. Balai Besar Penelitian Bioteknologi dan Sumberdaya Genetik. Diakses dari http://biogen.litbang.pertanian.go.id/

Martin, G.J, 1995, Ethnobotany, A People and Plants Conservation Manual.Chapman and Hall, London.

Maryadi. 2012. Studi Etnobotani Tumbuhan Obat di Desa Seriang Kecamatan Bedau Kabupaten Kapuas Hulu. Skripsi Fakultas Kehutanan. Universitas Tanjungpura. Pontianak.

Miles, Matthew B., and A. Michael Huberman, 2007, Qualitative Data Analysis (terjemahan), Jakarta: UI Press.

Mutaqin, A.Z., Astriani, W., Husodo, T., Partasasmita, R. 2018. Pemanfaatan Tumbuhan Untuk Beberapa Upacara Adat Oleh Masyarakat Desa Pangandaran Kecamatan Pangandaran Kabupaten Pangandaran. Jurnal Pro Life Vol. 5 No. 1.

Nurdin, B. V., and Ng, K. S. F. 2013. Local Knowledge of Lampung People in Tulang Bawang: An Ethnoecological and Ethnotechnological Study for Utilization and Conservation of Rivers. ProcediaSocial and Behavioral Sciences. 91: 113-119.

Patimah, 2010. Keanekaragaman Tumbuhan Obat di Kawasan Hutan Gunung Sinabung Kabupaten Karo Sumatera Utara. Universitas Sumetera Utara. Medan. 2 (1):120-128.

Rahyuni, Yniati, E., \& Pitopang, R. (2013). Kajian Etnobotani Tumbuhan Ritual Suku TAJIO di desa Kasimbar Kabupaten Paringi Mountong. Online Jurnal of Natural Science, 2(2), 46-54. 


\section{TECHNO: Vol. 09 (02) Oktober 2020}

Sardana A. , Hernawati J., Dharma N.G.G..Y, Nugroho A.E and Aliyah N. 2011. Potret Hutan Provinsi Kalimantan Barat (Figure of West Kalimantan Province). BPKH Wilayah III Pontianak. Pontianak.

Septiatin. 2008. Seri Tanaman Obat; Apotik Hidup dari Rempah-rempah, Tanaman Hias dan Tanaman Liar. Bandung: Yrama Widya.

Setyawan, A.D. 2010. Review: Biodiversity conservation strategy in a native perspective; a case study of shifting cultivation at the Dayaks of Kalimantan. Bioscience, Vol. 2, No. 2.

Sugiyono. 2007. Memahami Penelitian Kualitatif. Alfabeta. Bandung.

Supiandi, M.I., Susriyati, M., Siti, Z., Hendrikus, J., Benediktus, E. 2019. Ethnobotany of traditional medicinal plants used by Dayak Desa Community in Sintang, West Kalimantan, Indonesia. Biodiversitas Vol. 2 Number 5. DOI: 10.13057/biodiv/d200516

Takoy DM, Linda R, and Lovadi I, 2013. Tumbuhan Berkhasiat Obat Suku Dayak Seberuang di Kawasan Hutan Desa Ensabang Kecamatan Sepauk Kabupaten Sintang. Protobiont, Vol 2, No. 3, hal. 122-128.

Tapundu, A. S., \& Anam, S. (2015). Studi Etnobotani Tumbuhan Obat Pada Suku Seko Di Desa Tanah Harapan , Kabupaten Sigi , Sulawesi Tengah. Biocelebes, 9(2), 66-86.

Ting, Z., Chang-Hong, W., and Zheng-Tao, W. 2010. Chemical constituents and pharmacological actions of genus Peganum: research advances. Journal of International Pharmaceutical Research,37(5), 333-45.

Van Wyk, B-E., and Wink, M. 2004. Medicinal Plants of the World, 1st ed., Briza Publications, Pretoria (South Africa), p. 258.

Van Wyk, B-E., Van Oudtshoorn, B. and Gericke, N. 2002. Medicinal Plants of South Africa, 2nd ed., Briza Publications, Pretoria, pp. 202-203. 